\title{
Blindness, Short-sightedness, and Hirschberg's contextually ordered alternatives: a reply to Schlenker (2012)*
}

\author{
Giorgio Magri \\ SFL (CNRS, University of Paris 8)
}

\begin{abstract}
Magri (2009b,a, 2011) argues that scalar implicatures are blind to any contextual information. Schlenker (2012: sections 3 and 4) objects that contextual Blindness is too strong an assumption because of the contextually ordered alternatives documented in Hirschberg (1991). He thus submits that the computation of scalar implicatures is not blind but just contextually "short-sighted": it can ignore certain pieces of common knowledge, but it is not required to ignore all of it. In this paper, I show that Schlenker's proposal is a technical mistake: Short-sightedness is provably equivalent to Blindness under natural assumptions on the set of scalar alternatives. Short-sightedness thus provides no new ammunitions against Hirschberg's challenge. I then take a closer look at the challenge, through some initial evidence that contextually ordered alternatives are restricted and dependent on specific lexical choices. I conjecture that these choices share the property of introducing more logical structure than meets the eye, thus possibly providing the logical ordering required by Blindness (or the equivalent Short-sightedness). If this conjecture turns out to be correct, contextual ordering is never relevant to scalar implicatures, as indeed predicted by Blindness (or the equivalent Short-sightedness).
\end{abstract}

Keywords: scalar implicatures; oddness; contextually ordered alternatives; Gricean reasoning; common knowledge.

\section{Introduction}

In earlier work (Magri 2009b,a, 2011), I have defended an account for the oddness of sentence (1a) based on the following intuition. Just like any other existentially quantified sentence, this sentence (1a) triggers the scalar implicature that the universally quantified alternative (1b) is false, namely that not all Italians come from a warm country. Yet, common knowledge entails that all Italians come from the same country, rendering the existentially and universally quantified alternatives

* I would like to thank Gennaro Chierchia, Danny Fox, Salvatore Pistoia-Reda, Paolo Santorio, and Philippe Schlenker for useful comments. The paper also benefitted from comments and discussion at the Exhaustivity Workshop held at MIT on September 10th 2016. 
contextually equivalent. In conclusion, the oddness of sentence (1a) follows from a mismatch between the scalar implicature and common knowledge.

(1) a. \#Some Italians come from a warm country.

b. (All) Italians come from a warm country.

The formalization of this intuition runs into the following difficulty. Suppose that the algorithm for the computation of scalar implicatures had access to the common knowledge that all Italians come from the same country and thus to the information that the two alternatives (1a) and (1b) are contextually equivalent. No currently available implementation of that algorithm would then be able to derive the mismatching implicature needed to account for the oddness of sentence (1a). In my earlier work, I took this fact at face value and interpreted the oddness of sentence (1a) as evidence that the algorithm for the computation of scalar implicatures must be purely logical, namely blind to common knowledge. Section 2 reviews this Blindness hypothesis.

Schlenker (2012: sections 3 and 4) ${ }^{1}$ submits that Hirschberg's (1991) contextually ordered alternatives raise a challenge to Blindness. This challenge can be previewed as follows. Sentence (43a) triggers the scalar implicature that Mary dropped out of school after high school, namely that the alternative (43b) is false. Sentence (43b) instead does not trigger the scalar implicature that the alternative (43a) is false, as the sentence would otherwise mismatch with the piece of common knowledge that a college degree presupposes a high school degree.

(2) a. Mary has a high school degree.

b. Mary has a college degree.

It is tempting to correlate this asymmetry in scalar behavior with the fact that (43b) asymmetrically entails (43a). Yet, this entailment is contextual (it follows from common knowledge of the school system), not logical. In conclusion, such cases of contextually ordered alternatives seem to challenge the hypothesis that implicatures are blind to common knowledge and only sensitive to purely logical entailment. Section 3 reviews this challenge.

Schlenker thus suggests that the Blindness hypothesis is wrong and needs to be weakened. He puts forward a more moderate hypothesis: scalar implicatures are allowed to ignore certain pieces of common knowledge, as needed to derive the oddness of sentence (1a); yet, they are crucially not required to be blind to the entire common knowledge, thus possibly accommodating the common knowledge required to account for (43). Let me dub Schlenker's alternative proposal contextual

1 This paper is only concerned with the second part of Schlenker's paper (sections 3 and 4), not with the analysis of Maximize Presupposition developed in the first part (sections 1 and 2) of his paper-although my critical assessment of his principle of Fallibility in section 5 below extends to the first part of Schlenker's paper. 
Short-sightedness. Section 4 shows that Schlenker's Short-sightedness is actually provably equivalent to Blindness under natural assumptions on the set of scalar alternatives. I conclude that Schlenker's proposal is a technical mistake: Shortsightedness provides no new ammunitions against the challenge of contextually ordered alternatives and it is indeed a notational variant of Blindness.

Although Blindness and Short-sightedness are notational variants of each other, Schlenker submits that Short-sightedness has the advantage of being compatible with Gricean reasoning through a new pragmatic principle that he calls Fallibility. Section 5 reviews Schlenker's principle of Fallibility, briefly elaborates on the notion of "Griceanizability", and questions the Gricean credentials of Short-sightedness. Furthermore, I note that the challenge raised by contextually ordered alternatives extends to the (plain) meaning of sentences with only, whose interpretation ought to be hardwired in the semantics of the only operator and is thus independent of principles of Gricean pragmatics.

Section 6 takes a closer look at the challenge against Blindness (or, for that matter, the equivalent Short-sightedness) raised by Hirschberg's contextually ordered alternatives. If scalar implicatures had free access to contextual information, contextually ordered alternatives would be predicted to be freely available though proper manipulations of the common knowledge. Instead, I provide some initial evidence that contextually ordered alternatives are heavily restricted and dependent on specific lexical choices. I conjecture that these specific lexical choices share the property of introducing more logical structure than meets the eye, possibly providing the logical ordering required by Blindness (or Short-sightedness). For instance, sentence (43a) admits a degree construal whereby it says that Mary has a degree of education which is equal to or larger than the degree which corresponds to high school. Under this construal, its scalar implicature simply negates that she has a degree of education larger than that. The entailment between the base sentence and the negated alternative is purely logical, based on the ordering of degrees (of education). Under this analysis, the derivation of the implicature needs to know nothing about mundane facts concerning high schools and colleges. I conclude in section 7 with the conjecture that contextual ordering never plays any role in scalar behavior, as indeed predicted by Blindness (or the equivalent Short-sightedness).

\section{Blindness}

Subsections 2.1 and 2.2 sketch a framework for the description of scalar implicatures and thus set the background for the rest of the paper. Subsections 2.3 and 2.4 then summarize the Blindness hypothesis that was argued in Magri (2009b,a, 2011) to be needed to formalize the intuition that a sentence such as (1a) sounds odd because of the scalar implicature that not all Italians come from a warm country. 


\subsection{Strengthened meaning}

Let me denote by $[\llbracket \varphi]]^{\mathrm{c}}$ the strengthened meaning of a base sentence $\varphi$ relative to the common knowledge $\mathrm{c}$ available in the context of utterance, namely the plain meaning of $\varphi$ enriched with the scalar implicatures triggered by $\varphi$ in a context characterized by the common knowledge $c^{2}{ }^{2}$ The crucial role played by common knowledge in what follows is explicitly recognized right from the outset, by letting it figure as a parameter $\mathbf{c}$ in the definition of the strengthened meaning. ${ }^{3}$ To illustrate, the base sentence $\varphi_{\text {most }}$ in (3a) triggers the scalar implicature that John stopped short of doing the entire homework. Equivalently, it triggers the implicature $\neg \psi_{\text {all }}$ that the alternative $\psi_{\text {all }}$ in (3b) is false. Hence, the strengthened meaning $\left.\left[\llbracket \varphi_{\text {most }} t\right]\right]^{\mathrm{c}}$ of the base sentence $\varphi_{\text {most }}$ is the conjunction of $\varphi_{\text {most }}$ with the negation $\neg \psi_{\text {all }}$ of the alternative $\psi_{\text {all }}$, as indicated in (3). ${ }^{4}$

$$
\begin{aligned}
& {\left[\llbracket \varphi_{\text {most }} \rrbracket\right]^{c}=\varphi_{\text {most }} \wedge \neg \psi_{\text {all }}} \\
& \text { a. John did most of the homework. } \\
& \text { b. John did all of the homework. } \\
& \text { c. John did some of the homework. }
\end{aligned}
$$

$$
\begin{array}{r}
=\varphi_{\text {most }} \\
=\psi_{\text {all }} \\
=\psi_{\text {some }}
\end{array}
$$

In general, the strengthened meaning $\llbracket[\varphi \rrbracket]]^{\mathrm{c}}$ of a base sentence $\varphi$ is described as the conjunction of the plain meaning of $\varphi$ with the negation of a bunch of alternatives $\psi_{1}, \psi_{2}, \ldots$, as in (4a). It is convenient to collect all these excluded alternatives in a set usually denoted by $\operatorname{Excl}^{\mathrm{c}}(\varphi)$, as in (4b). The dependence of the strengthened meaning $[[[\cdot]]]^{\mathrm{c}}$ on the common knowledge c percolates down to the set $\operatorname{Excl}^{\mathrm{c}}(\cdot)$ of excluded alternatives.

$$
\llbracket \llbracket \varphi \rrbracket]^{\mathrm{c}} \stackrel{(a)}{=} \varphi \wedge \neg \psi_{1} \wedge \neg \psi_{2} \wedge \ldots \stackrel{(b)}{=} \varphi \wedge \bigwedge_{\psi \in \operatorname{Excl}^{\mathrm{c}}(\varphi)} \neg \psi
$$

The issue of the proper description of the strengthened meaning $[\llbracket \varphi]]^{c}$ thus boils down to the issue of the proper description of the set $\operatorname{Excl}^{\mathbf{C}}(\varphi)$ of excluded alternatives (relative to the common knowledge $\mathbf{c}$ ). The latter issue is delicate. To illustrate, the alternative $\psi_{\text {all }}$ in (3b) must belong to the set $\operatorname{Excl}^{\mathbf{C}}\left(\varphi_{\text {most }}\right)$, in order to derive the intended strengthened meaning. Yet, the alternative $\psi_{\text {some }}$ in $(3 \mathrm{c})$ cannot belong to the set $\operatorname{Excl}^{\mathrm{C}}\left(\varphi_{\text {most }}\right)$, as the strengthened meaning of $\varphi_{\text {most }}$ would otherwise be condemned to be a contradiction. What is the relevant difference

2 The term strengthened meaning is non-committal relative to the debate concerning the pragmatic versus grammatical nature of scalar implicatures (Horn 2005; Chierchia, Fox \& Spector 2012).

3 I assume that $\mathbf{c}$ is the set of possible worlds consistent with the common knowledge currently available in the context of utterance. Since the examples considered throughout the paper are all elementary, this parameter $\mathbf{c}$ will never be used in the plain semantics. It will only be relevant for the definition of the excluded alternatives; see (5) and (6) below.

4 With abuse of notation, I use the same symbol $\varphi, \psi, \ldots$ for both a sentence and its plain meaning. 
between these two alternatives $\psi_{\text {all }}$ and $\psi_{\text {some }}$ that can be exploited in the definition of the set $\operatorname{Excl}^{\mathrm{c}}\left(\varphi_{\text {most }}\right)$ of excluded alternatives? Two answers have been explored in the literature, summarized in the next subsection. This discussion will be relevant for the developments in subsection 4.2.

\subsection{Excluded alternatives}

One characterization of the set of excluded alternatives follows from the logics of the (neo)-Gricean approach to scalar implicatures (Horn 1972; Geurts 2010). According to this approach, a scalar implicature arises through the comparison of the speaker's utterance $\varphi$ with an alternative $\psi$ that the speaker could have uttered instead and that would have counted as a "better" utterance. If conversation is construed as a game that maximizes information exchange between speaker and addressee, $\psi$ counts as "better" than $\varphi$ in case $\psi$ would have provided more information than $\varphi$, namely $\psi$ asymmetrically entails $\varphi$. This line of reasoning leads to the definition (5) of the set $\operatorname{Excl}^{\mathrm{c}}(\varphi)$ of excluded alternatives.

(5) $\operatorname{Excl}^{\mathrm{C}}(\varphi)$ consists of those scalar alternatives $\psi$ of $\varphi$ such that $\psi$ asymmetrically entails $\varphi$ relative to c, namely $\psi \cap \mathrm{c} \subsetneq \varphi \cap \mathrm{c}$.

According to this approach, the relevant difference between the two alternatives $\psi_{\text {all }}$ and $\psi_{\text {some }}$ in (3) is that only the former asymmetrically entails $\psi_{\text {most }}$, so that only the former ends up in the corresponding set $\operatorname{Excl}^{\mathbf{c}}\left(\psi_{\text {most }}\right)$ of excluded alternatives.

An alternative characterization of the set of excluded alternatives has been endorsed within the grammatical approach to scalar implicatures (Chierchia 2004; Chierchia et al. 2012; Spector to appear). According to this approach, implicatures are derived not through pragmatic, extra-grammatical reasoning, but through a syntactically realized covert operator akin to only (I will come back to this point in subsection 2.4). From this perspective, excluded alternatives don't need to be "better" than the base sentence, as long as they are not "harmful". More precisely, excluded alternatives do not need to asymmetrically entail the base sentence. Instead, it suffices that their negation does not contradict the base sentence, leading to the definition (6) of the set $\operatorname{Excl}^{\mathrm{c}}(\varphi)$ of excluded alternatives. ${ }^{5}$

5 More precisely, it suffices that excluded alternatives do not yield a contradictory strengthened meaning $\llbracket \llbracket \varphi \rrbracket \rrbracket]^{c}$. Condition (6), as it stands, does not ensure that. In fact, suppose that $\varphi$ comes with two alternatives $\psi_{1}$ and $\psi_{2}$ whose negation does not contradict $\varphi$, namely $\varphi \cap \mathbf{c} \cap \neg \psi_{1} \neq \emptyset$ and $\varphi \cap \mathbf{c} \cap \neg \psi_{2} \neq \emptyset$. Hence, both $\psi_{1}$ and $\psi_{2}$ belong to the corresponding set $\operatorname{Excl}^{\mathbf{c}}(\varphi)$ of excluded alternatives according to definition (6). Yet, the strengthened meaning $\llbracket \varphi \|^{\mathrm{c}}=\varphi \wedge \neg \psi_{1} \wedge \neg \psi_{2}$ could still be a contradiction, namely it could still happen that $\varphi \cap \mathbf{c} \cap \neg \psi_{1} \cap \neg \psi_{2}=\emptyset$. The formulation in (6) is therefore too weak and it has indeed been carefully refined in the literature in a number of ways (see Spector to appear for a review). I ignore these refinements because all the cases considered in this paper involve a unique alternative, so that the refinements considered in the literature turn out to 
(6) $\operatorname{Excl}^{\mathrm{c}}(\varphi)$ consists of those scalar alternatives $\psi$ of $\varphi$ such that the negation of $\psi$ does not contradict $\varphi$ relative to c, namely $\varphi \cap \mathrm{c} \cap \neg \psi \neq \emptyset$.

According to this approach, the relevant difference between the two alternatives $\psi_{\text {all }}$ and $\psi_{\text {some }}$ in (3) is that only the former can be negated without contracting $\psi_{\text {most }}$, so that only the former ends up in the corresponding set $\operatorname{Excl}^{\mathbf{C}}\left(\psi_{\text {most }}\right)$ of excluded alternatives.

\subsection{Oddness filter}

I am now ready to go back to the problem of accounting for the oddness of sentences such as (1a), repeated as $\varphi_{\text {some }}$ in (7a).

(7) a. \#Some Italians come from a warm country.

$$
\begin{gathered}
=\varphi_{\text {some }} \\
=\psi_{\text {all }}
\end{gathered}
$$

b. (All) Italians come from a warm country.

As anticipated, I want to capture the intuition that $\varphi_{\text {some }}$ sounds odd because it triggers the scalar implicature that the alternative $\psi_{\text {all }}$ in (7b) is false, contradicting the common knowledge that all Italians come from the same country. I thus need an oddness filter which takes into account the strengthened meaning, as in (8).

(8) Oddness filter: preliminary formulation.

A sentence $\varphi$ sounds odd relative to the actual common knowledge $\mathrm{c}_{\text {actual }}$ provided $[[[\varphi]]]] \mathrm{c}^{\mathrm{c}} \cap \mathrm{c}_{\text {actual }}=\emptyset$.

This preliminary formulation (8) of the oddness filter purposely features a free parameter, namely the common knowledge $c$ used in the computation of the strengthened meaning $[[[\varphi]]]]^{\mathrm{c}}$ of the odd sentence $\varphi$. A natural choice would be to set this parameter $\mathbf{c}$ equal to the actual common knowledge $\mathbf{c}_{\text {actual }}$, yielding the reformulation (9) of the oddness filter. This formulation is plausibly purely pragmatical. In fact, the relevant meaning for pragmatics ought to be the strengthened meaning relative to the actual common knowledge. And it makes sense for pragmatics to require the relevant meaning of the speaker's utterance not to be a contextual contradiction. Let me thus refer to (9) as the vanilla formulation of the oddness filter.

(9) Oddness filter: vanilla formulation.

A sentence $\varphi$ sounds odd relative to the actual common knowledge $\mathrm{c}_{\text {actual }}$ provided $[[[\varphi]]]^{\mathrm{C}} \cap \mathrm{c}_{\mathrm{actual}}=\emptyset$ where $\mathrm{C}=\mathrm{C}_{\mathrm{actual}}$.

Unfortunately, this vanilla formulation fails at accounting for the oddness of the sentence $\varphi_{\text {some }}$ in (7a). In fact, $\varphi_{\text {some }}$ and $\psi_{\text {all }}$ are equivalent relative to the actual common knowledge $\mathbf{c}_{\text {actual }}$ (because $\mathbf{c}_{\text {actual }}$ entails that all Italians come from the be equivalent to the simplified formulation (6). 
same country). Hence, $\psi_{\text {all }}$ cannot belong to the set $\operatorname{Excl}^{\mathrm{c}_{\text {actual }}}\left(\varphi_{\text {some }}\right)$ of alternatives excluded by $\varphi_{\text {some }}$ relative to the actual common knowledge $\mathrm{c}_{\text {actual }}$, no matter which of the two definitions (5) or (6) of excluded alternatives is adopted. Thus, the strengthened meaning $[[\varphi]]]]^{c_{a c t u a l}}$ computed relative to the actual common knowledge $\mathbf{C}_{\text {actual }}$ is not a contextual contradiction.

In Magri (2009b,a, 2011), I took this fact at face value and proposed a reformulation of the oddness filter where the strengthened meaning is computed not relative to the actual common knowledge $c_{\text {actual }}$ but relative to the entire set $\mathscr{W}$ of possible worlds, leading to the revised formulation (10) of the oddness filter. The boxed condition in (10) says that the strengthened meaning which enters into the oddness filter is completely blind to any piece of common knowledge. This boxed condition is thus dubbed Blindness.

\section{(10) Oddness filter: blind formulation.}

A sentence $\varphi$ sounds odd relative to the actual common knowledge $\mathrm{c}_{\text {actual }}$ provided $[[[\varphi]]]^{\mathrm{c}} \cap \mathrm{c}_{\text {actual }}=\emptyset$ where $\mathrm{c}=\mathscr{W}=$ the set of all possible worlds .

The logical strengthened meaning of the sentence $\varphi_{\text {some }}$ is $\left[\left[\left[\varphi_{\text {some }}\right]\right]\right]^{\mathrm{c}=\mathscr{W}}=\varphi_{\text {some }} \wedge$ $\neg \psi_{\text {all }}$, namely the proposition that some but not all Italians come from a warm country. This proposition is indeed a contextual contradiction, given the common knowledge that all Italians come from the same country. The blind oddness filter thus succeeds at predicting oddness. The price to pay is that the pragmatic justification of the vanilla formulation of the oddness filter does not extend to the blind reformulation. A different justification is thus needed, sketched in the next subsection.

\subsection{Overt only}

The sentence Some Italians come from a warm country in (7a) remains odd when only is added, as in (11). Plausibly, scalar implicatures are not at stake here. Instead, it is tempting to derive oddness from the fact that, because of only, this sentence says that some but not all Italians come from a warm country, which contradicts the piece of common knowledge that all Italians come from the same country. Let's formalize this intuition.

(11) \#Only some Italians come from a warm country.

Abstracting away from a number of (hopefully orthogonal) subtleties (Beaver $\&$ Clark 2008), let me assume that only takes a prejacent sentence $\varphi$ and returns its conjunction with the negation of a bunch of alternatives. Again, it is convenient to collect these alternatives in a set of excluded alternatives. Fox (2007) observes that the strengthened meaning of a sentence such as John did most of the homework in 
(3a) can be paraphrased by the variant with only (associating with the scalar item), namely John only did most of the homework. Hence, the set of excluded alternatives in the semantics of only plausibly coincides with the set of excluded alternatives in the definition of the strengthened meaning, yielding (12) in analogy with (4).

$$
[[\operatorname{only}]](\varphi)=\varphi \wedge \bigwedge_{\psi \in \operatorname{Excl}^{\mathrm{c}}(\varphi)} \neg \psi
$$

The semantics of only thus becomes sensitive to the parameter c which enters into the definition of the set $\mathrm{Excl}^{\mathrm{c}}$ of excluded alternatives. It would be natural to set this parameter $\mathbf{c}$ in (12) equal to the actual common knowledge $\mathbf{c}_{\text {actual }}$. Yet, this assumption would fail to predict the (plain) meaning of sentence (11) to be a contextual contradiction, for the by now familiar reason: $\mathbf{c}_{\text {actual }}$ entails that all Italians come from the same country and thus prevents the alternative $\psi_{\text {all }}$ from counting as excludable relative to the prejacent $\varphi_{\text {some }}$, no matter which definition of excludability is adopted. I conclude that the knowledge $\mathrm{c}$ in the set of excluded alternatives in the plain semantics (12) of only is different from the actual common knowledge $\mathbf{c}_{\text {actual. }}{ }^{6}$

The definition (4) of the strengthened meaning and the semantics (12) for only are completely analogous, in order to capture the fact that the strengthened meaning of a sentence can be paraphrased by adding only. Various authors have indeed suggested that the strengthened meaning is actually brought about through a covert only, not through pragmatic, extra-grammatical reasoning (Fox 2007; Chierchia et al. 2012). As we have just seen, the set of excluded alternatives of overt only is blind to the actual common knowledge $\mathrm{c}_{\text {actual }}$ - possibly a general property of the syntax/semantics interface (Fox 2000). It then makes sense to assume the same to be the case for covert only. The strengthened meaning is therefore blind to common knowledge and the oddness filter cannot but check the contextual contraddictorinness of this blind strengthened meaning, as indeed assumed in (10).

\section{The challenge raised by contextually ordered scales}

Schlenker submits that Hirschberg's (1991) contextually ordered alternatives are a challenge for Blindness. Although Schlenker's specific formulation of the challenge

6 This conclusion is threatened by the following alternative account. Assume instead that the set of excludable alternatives for only in (12) is computed relative to the actual common knowledge $\mathbf{c}_{\text {actual }}$. Thus, the alternative $\psi_{\text {all }}$ does not belong to the set of excludable alternatives $\operatorname{Excl}^{\mathrm{c}}$ actual $\left(\varphi_{\text {some }}\right)$ of the prejacent $\varphi_{\text {some, }}$, independently of the definition of excludability. The oddness of sentence (11) is thus due not to a contextual contradiction but to the fact that only is vacuous because its set of excluded alternatives is empty. In other words, sentence (11) is ruled out by the same general constraint that bans the vacuous occurrence of only in a sentence such as \#Only every boy arrived. Yet, this alternative line of explanation fails for cases with multiple alternatives (for the full argument, see Magri 2011: section 3). 
is moot (subsection 3.1), the challenge is real (subsection 3.2) and will be discussed in the rest of the paper.

\subsection{Contextually equivalent alternatives}

Schlenker asks us to consider a scenario where the actual common knowledge $\mathbf{c}_{\text {actual }}$ entails the proposition (13a), say because it has been established through some preceding discourse. The actual common knowledge $\mathbf{c}_{\text {actual }}$ of course also entails a wealth of additional information, including the proposition (13b) concerning typical college admittance requirements. Against this background, Schlenker notes the oddness contrast in (14). An analogous contrast holds for the corresponding sentences with overt only.

(13) The actual common knowledge $\mathbf{c}_{\text {actual }}$ entails:

a. That every employee in my company is either a janitor with no school degree or else a programmer with a college degree.

b. That a college degree presupposes a high school degree.

a. \#My colleague Mary has a high school degree.

$$
\begin{array}{r}
=\varphi_{\text {high-school }} \\
=\varphi_{\text {college }}
\end{array}
$$

b. My colleague Mary has a college degree.

Assume that the oddness of sentence $\varphi_{\text {high-school }}$ in (14a) is due to a mismatch between the piece of common knowledge (13a) and the scalar implicature $\neg \varphi_{\text {college }}$ that Mary lacks a college degree through the strengthened meaning (15a). The computation of this mismatching implicature must be blind to the piece of common knowledge (13a). Since Blindness does not make any distinctions among different pieces of common knowledge, the computation of scalar implicatures is also blind to the piece of common knowledge (13b). Sentence $\varphi_{\text {college }}$ in $(14 \mathrm{~b})$ is therefore incorrectly predicted to be odd as well, because of a mismatch between the piece of common knowledge (14b) and the scalar implicature $\neg \varphi_{\text {high-school }}$ that Mary lacks a high school degree through the strengthened meaning $(15 b)$.
a. $\left[\left[\left[\varphi_{\text {high-school }}\right]\right]\right]^{\mathscr{W}}=\varphi_{\text {high-school }} \wedge \neg \varphi_{\text {college }}$
requires ignorance of (13a)
b. $\left[\left[\left[\varphi_{\text {college }}\right]\right]\right]^{\mathscr{W}}=\varphi_{\text {college }} \wedge \neg \varphi_{\text {high-school }}$
requires ignorance of (13b)

In conclusion, the symmetric formalism (15) is unable to capture the oddness asymmetry in (14). Schlenker (p. 421) diagnoses that "this symmetry problem cannot be solved without access to contextual knowledge-which seems to contradict the essence of Magri's idea of blind implicatures". But this diagnosis seems to me flawed. In fact, the crucial property of Schlenker's example (13)-(14) is that the two alternatives $\varphi_{\text {high-school }}$ and $\varphi_{\text {college }}$ are contextually equivalent in his scenario: in 
every world in $\mathbf{c}_{\text {actual }}$, one of the two alternatives is true if and only if the other one is true as well. Hence, access to common knowledge could hardly help accounting for the asymmetry in (14) which therefore has nothing to say about Blindness.

\subsection{Contextually asymmetrically entailing alternatives}

Although Schlenker's argument (13)-(14) against Blindness is moot, the following simpler variant does raise a serious challenge. Out of the blue, sentence $\varphi_{\text {high school }}$ in (16a) triggers the scalar implicature that Mary dropped out of school after high school and thus lacks a college degree. That is indeed the meaning of the corresponding sentence with overt only. In other words, the strengthened meaning of sentence $\varphi_{\text {high school }}$ is (16b), which features the negation of the alternative $\varphi_{\text {college }}$.

a. Mary has a high school degree.

$=\varphi_{\text {high school }}$

b. $\quad\left[\left[\left[\varphi_{\text {high school }}\right]\right]\right]=\varphi_{\text {high school }} \wedge \neg \varphi_{\text {college }}$

a. Mary has a college degree.

b. $\left.\quad\left[\left[\varphi_{\text {college }}\right]\right]\right] \neq \varphi_{\text {college }} \wedge \neg \varphi_{\text {high school }}$

$=\varphi_{\text {college }}$

The case of sentence (17a) is rather different. It does not in any way trigger the scalar implicature that Mary skipped high school. Indeed, the meaning of the corresponding sentence with overt only does not in any way single out Mary as an exception to the standard school system. In other words, the strengthened meaning of sentence $\varphi_{\text {college }}$ does not feature the negation of the alternative $\varphi_{\text {high school }}$, as indicated in (17b).

What could be responsible for the asymmetry between the two sentences $\varphi_{\text {high school }}$ and $\varphi_{\text {college }}$ displayed in (16)-(17)? It is natural to attribute that asymmetry to the fact that college presupposes high school. In other words, $\varphi_{\text {college }}$ asymmetrically entails $\varphi_{\text {high school }}$. This entailment holds relative to common knowledge on the actual school system. From a logical perspective, no entailment relationship whatsoever holds between $\varphi_{\text {high school }}$ and $\varphi_{\text {college }}$. These two alternatives are thus an instance of what Hirschberg (1991) calls contextually ordered alternatives. The obvious problem for Blindness is that it predicts the scalar behavior of the two alternatives to track their logical independence rather than their contextual asymmetry. Blindness thus incorrectly predicts the two alternatives to display a perfectly analogous scalar behavior: either neither of the two triggers the implicature that the other is false, whereby $\varphi_{\text {high school }}$ lacks the attested implicature; or else both alternatives trigger the implicature that the other is false, whereby $\varphi_{\text {college }}$ displays an unattested implicature that should turn it into a contextual contradiction. Hirschberg's contextually ordered alternatives defy the very idea of Blindness.

Obviously, no threat against contextual Blindness would arise if the asymmetry in the scalar behavior of $\varphi_{\text {high school }}$ and $\varphi_{\text {college }}$ were to follow from some other properties than their contextual asymmetric entailment relationship. Indeed, the 
challenge for Blindness only stems from the assumption that it is the contextual asymmetric entailment that is responsible for the asymmetric scalar behavior. This assumption leads to the prediction that, if we were to tamper with the out-of-the-blue common knowledge to the effect of rendering the two alternatives $\varphi_{\text {high school }}$ and $\varphi_{\text {college }}$ contextually equivalent (rather than contextually asymmetrically entailing), the asymmetry in their scalar behavior should disappear. Schlenker's example (13)(14) is interesting because it shows that this prediction is not borne out. It thus lends support to the hypothesis that contextual ordering plays no role in the asymmetric scalar behavior in (16)-(17), as indeed predicted by Blindness. In other words, Schlenker's example actually takes some edge off the apparently straightforward challenge to Blindness raised by Hirschberg's contextually ordered scales. I will revisit this issue in subsection 6.2 .

\subsection{A digression on the contextual nature of the existential import of univer- sal quantifiers}

Schlenker submits that the challenge to Blindness based on contextually ordered alternatives just reviewed extends to existentially quantified sentences. His starting point is the observation that 'there are examples in which 'Every $P, Q$ ' does not carry an implication, let alone a presupposition, that there are $P$-individuals." He provides two such examples and concludes that a universally quantified sentence 'Every $P$, $Q$ ' simply requires $P$ to be a subset of $Q$, leaving open the possibility that this subset condition be satisfied through the emptiness of $P$. Against this background, Schlenker's submits that the challenge to Blindness raised by contextually ordered alternatives re-presents itself with the two alternatives $\varphi_{\text {some }}, \varphi_{\text {all }}$ in (19).

(18) The actual common knowledge $c_{\text {actual }}$ entails:

a. That all Italians come from the same country.

b. That Italians exist.

a. \#Some Italians come from a warm country. $=\varphi_{\text {some }}$

b. (All) Italians come from a warm country.

$=\varphi_{\text {all }}$

The sentence $\varphi_{\text {some }}$ in (19a) sounds odd because it triggers the scalar implicature $\neg \varphi_{\text {all }}$ through the strengthened meaning in (20a). The computation of this mismatching implicature must be blind to the piece of common knowledge (18a). Since Blindness does not make any distinctions among different pieces of common knowledge, the computation of scalar implicatures is also blind to the piece of common knowledge (18b). If the sentence $\varphi_{\text {all }}$ in (19b) does not semantically presuppose the existence of Italians, it can trigger the scalar implicature $\neg \varphi_{\text {some }}$ through the strengthened 
meaning (20b). As Schlenker observes, the conjunction $\varphi_{\text {all }} \wedge \neg \varphi_{\text {some }}$ thus derived is equivalent to There exist no Italians. Sentence (19b) is thus incorrectly predicted to be odd because of a mismatch with the piece of common knowledge (18b).
a. $\left[\left[\left[\varphi_{\text {some }}\right]\right]\right]^{\mathscr{W}}=\varphi_{\text {some }} \wedge \neg \varphi_{\text {all }}$
requires ignorance of (18a)
b. $\left.\quad\left[\left[\varphi_{\text {all }}\right]\right]\right]^{\mathscr{W}}=\varphi_{\text {all }} \wedge \neg \varphi_{\text {some }}$
requires ignorance of $(18 b)$

Schlenker submits that the challenge is specific to Blindness because "for standard [namely non-blind] accounts of implicatures, this observation need not pose a serious difficulty; they may simply posit that contextual knowledge [such as (18b)] can be accessed when checking that the pair $\langle$ 'Some $P, Q$ ', 'Every $P, Q$ '〉 stands in the appropriate relation of asymmetric entailment."

The presuppositional nature of the existential import of universal quantifiers has been the subject of a very large literature, with certain authors (such as Russell 1904; Strawson 1950; Hart 1951; de Jong \& Verkuyl 1991; Horn 1997; Geurts 2007) supporting a semantic/presuppositional treatment while others (such as Lappin \& Reinhart 1988 and Abusch \& Rooth 2004) have explored alternative non-presuppositional approaches. Since Schlenker does not address this literature, it is hard to interpret his two isolated examples within the context of the larger debate. Be that as it may, this version of Schlenker's challenge against Blindness crucially hinges on the assumption that, in cases such as (19), it is common knowledge (18b) which crucially provides the correct relationship between the universal and the existential alternatives. Yet, the contrast in (21) from Pistoia-Reda (to appear) speaks against this assumption. Common knowledge plausibly does not entail the existence of Swedish matadors. Indeed, sentence $(21 \mathrm{~b})$ is judged acceptable but false (in accordance with previous literature; Lappin \& Reinhart 1988), precisely because there are no Swedish matadors in the actual word.

(21) a. \#Some Swedish matadors come from a cold country.

b. Some Swedish matadors know Latin

Pistoia-Reda notes that sentence (21a) is instead judged odd, just as the original sentence (19a). Plausibly, the existentially quantified sentences (19a) and (21a) are both odd because of the implicature that the corresponding universally quantified alternative is false. But the analogy breaks down: only common knowledge on Italians, not the one on Swedish matadors, provides "the appropriate relation of asymmetric entailment" between the alternatives. This suggests that it is not common knowledge that is at stake here. In the rest of this paper, I will thus put aside this issue of the proper analysis of existential import. 


\section{Short-sightedness}

How can we tackle the challenge to Blindness raised by Hirschberg's contextually ordered alternatives? Schlenker (p. 421) diagnoses that "the problem is that Magri's account is in a way 'too' blind: it has insufficient access to contextual knowledge." He thus proposes an alternative which is 'less' blind and that I will therefore dub contextual Short-sightedness. Yet, this section shows that, under natural assumptions on the set of alternatives, Schlenker's Short-sightedness is provably equivalent to Blindness: they are a notational variant of each other. I conclude that Schlenker's proposed solution is a technical mistake: Short-sightedness offers no new ammunitions against the challenge of contextually ordered alternatives. Section 6 will thus take a closer look at the challenge.

\subsection{Weakening Blindness to short-sightedness}

The actual common knowledge $\mathbf{c}_{\text {actual }}$ provides a wealth of information about Italy and Italians, some of which is listed in (22).

(22) The actual common knowledge $\mathbf{c}_{\text {actual }}$ entails:

a. That all Italians come from the same country.

b. That Italian food is way better than French food.

The Blindness condition boxed in (10), repeated below, says that, when checking the oddness of the sentence $\varphi=$ Some Italians come from a warm country in (7a), we consider its strengthened meaning $[\llbracket \varphi \rrbracket]]^{\mathrm{c}=\mathscr{W}}$ relative to the entire set $\mathscr{W}$ of possible worlds. This means that each one of the various pieces of common knowledge listed in (22) is wiped out.

(10) Oddness filter: blind formulation (repeated).

A sentence $\varphi$ sounds odd relative to the actual common knowledge $\mathrm{c}_{\text {actual }}$ provided $\llbracket[\varphi]]]^{\mathrm{C}} \cap \mathrm{c}_{\text {actual }}=\emptyset$ where $\mathrm{c}=\mathscr{W}=$ the set of all possible worlds .

Schlenker (2012: section 4) suggests that this is overkilling and thus advocates a more moderate approach. He correctly notices that, in order for the implicature-based account of the oddness of our sentence $\varphi=$ Some Italians come from a warm country to go through, we only need the strengthened meaning to be blind to the piece of common knowledge (22a). Other pieces of common knowledge, such as (22b), are inoffensive. Since they are inoffensive, there is no reason why they need to be wiped out as well. Schlenker therefore replaces the blind oddness filter (10) with the variant in (23). As he explains, "the basic idea is that if any weakening of a (global or local) 
context gives rise to an implicature-whether global or embedded-which contradicts the assertive component of the clause that triggers it, we obtain a misleading implicature, and the sentence is deviant." (p. 423).

\section{(23) Oddness filter: short-sighted formulation.}

A sentence $\varphi$ sounds odd relative to the actual common knowledge $\mathrm{c}_{\text {actual }}$ provided $[[[\varphi]]]]^{\mathbf{c}} \cap \mathbf{c}_{\text {actual }}=\emptyset$ for some $\mathbf{c}$ such that $\mathscr{W} \supseteq \mathbf{c} \supseteq \mathbf{c}_{\text {actual }}$.

The two filters (10) and (23) only differ for the boxed conditions. In particular, both proposals assume that the common knowledge $\mathrm{c}$ used to compute the strengthened meaning relevant for the oddness filter is not the actual common knowledge $\mathbf{c}_{\text {actual }}$ but a weakening $\mathrm{c}$ thereof. The two proposals differ because Schlenker does not require this weakening c to be the weakest weakening, namely the entire set $\mathscr{W}$ of all possible worlds. In particular, while $\mathscr{W}$ differs from the actual common knowledge $\mathbf{c}_{\text {actual }}$ because it contains worlds where each of the propositions in (22) fails, Schlenker's weakening c could still support, say, (22b), while allowing for possible words where (22a) fails. In conclusion, Schlenker's replaces Blindness with contextual Short-sightedness.

\subsection{Short-sightedness is equivalent to Blindness}

As anticipated in subsection 2.2, a crucial choice point in the definition of the strengthened meaning concerns the proper definition of the set of excluded alternatives: in terms of asymmetric entailment, as in (5); or else in terms of noncontradictoriness, as in (6). As this distinction will be relevant throughout this subsection, let me replace the ambiguous notation $[[[\cdot]]]$ and Excl used so far with the notation $\left.[[[\cdot]]]_{\mathrm{AE}},[[\cdot \cdot]]\right]_{\mathrm{NC}}$ and $\operatorname{Excl}_{\mathrm{AE}}, \operatorname{Excl}_{\mathrm{NC}}$, which disambiguates whether asymmetric entailment (AE) or non-contradictoriness (NC) is used in the definition of the set of excluded alternatives and thus in the computation of the strengthened meaning.

Schlenker is not explicit on which one of these two options he endorses. His discussion seems to assume excluded alternatives to be defined in terms of asymmetric entailment. Yet, he (p. 418) incorrectly attributes the choice of asymmetric entailment over non-contradictoriness to Magri (2011), suggesting that he is sticking with that choice only for ease of comparison with my earlier work. That is not correct: Magri (2009b,a, 2011) unequivocally advocates the definition of excluded alternatives in terms of non-contradictoriness. Indeed, I submit that the literature has nowadays reached a consensus that the definition in terms of asymmetric entailment is too restrictive and needs to be replaced by a definition in terms of non-contradictoriness (see Spector to appear).

Be that as it may, this subsection proves that the short-sighted oddness filter (23) is equivalent to the blind oddness filter (10), irrespectively of whether excludability is 
defined in terms of non-contradictoriness or asymmetric entailment. This equivalence holds under mild and natural assumptions on the set of scalar alternatives and under no assumptions whatsoever on the actual common knowledge $\mathbf{c}_{\text {actual }}$.

\subsubsection{When exclusion is defined through non-contradictoriness}

Let me start with the case where the strengthened meaning used in the oddness filter is $[[[\varphi]]]_{\mathrm{NC}}$, namely it is defined in terms of non-contradictoriness. In this case, Short-sightedness and Blindness are equivalent without any additional assumptions on the set of scalar alternatives, as stated in (24). The equivalence (24) is mentioned by Schlenker in subsection 4.3.4 of his NALS paper. Yet, he informs me (p.c. by email of 01/18/2012) that he decided not to include that subsection in the submitted version of the paper but added it at the end of the NALS review process. The present paper therefore represents the first time that the equivalence (24) undergoes peer reviewing.

\begin{tabular}{|lll|}
\hline Blindeness: & Short-sightedness: \\
{$[[[\varphi]]]_{\mathrm{NC}}^{\mathrm{c}} \cap \mathrm{c}_{\text {actual }}=\emptyset$} & $\Longleftrightarrow$ & $[[\varphi]]]_{\mathrm{NC}}^{\mathrm{c}} \cap \mathrm{c}_{\text {actual }}=\emptyset$ \\
with $\mathrm{c}=\mathscr{W}$ & for some $\mathrm{c} \supseteq \mathrm{c}_{\text {actual }}$ \\
\hline
\end{tabular}

In fact, the implication $\Longrightarrow$ trivially follows from the fact that $\mathrm{c}$ is existentially quantified over by Short-sightedness and subject only to the condition that $\mathbf{c} \supseteq \mathbf{c}_{\text {actual }}$. The latter condition is obviously satisfied by the position $c=\mathscr{W}$ considered by Blindness. The reverse implication $\Longleftarrow$ follows from the obvious property (25) of the set $\operatorname{Excl}_{\mathrm{NC}}^{\mathrm{c}}$ of excluded alternatives defined in terms of non-contradictoriness: it is monotone relative to the common knowledge $\mathrm{c}$, in the sense that it cannot shrink when the world set c grows. ${ }^{7}$

(25) For any two set of worlds $\mathbf{c}^{\prime}$ and $\mathbf{c}^{\prime \prime}$, for any alternative $\psi$ :

If: $\quad \mathbf{c}^{\prime} \subseteq \mathbf{c}^{\prime \prime}$

Then: $\quad \psi \in \operatorname{Excl}_{\mathrm{NC}}^{\mathrm{c}^{\prime}}(\varphi)$ entails that $\psi \in \operatorname{Excl}_{\mathrm{NC}}^{\mathrm{c}^{\prime \prime}}(\varphi)$

It follows that any alternative $\psi$ negated by the short-sighted strengthened meaning $[[[\varphi]]]_{\mathrm{NC}}^{\mathrm{c}}$ is also excluded by the blind strengthened meaning $[[\varphi \varphi]]_{\mathrm{NC}}^{\mathrm{c}=\mathscr{W}}$. If the former strengthened meaning is a contextual contradiction, the latter is a contextual contradiction as well. The equivalence in (24) is therefore established.

As reviewed in section 3, contextually ordered alternatives challenge Blindness. Let me show that Short-sightedness fares no better when the alternatives are defined

7 As explained in footnote 5, the specific definition of $\operatorname{Excl}_{\mathrm{NC}}$ provided in (6) is too weak, because it looks at non-contradictoriness of the negation of each individual alternative by itself. This definition needs to be replaced with a more careful restatement, which looks at the non-contradictoriness of the conjunction of the negations of an entire bunch of alternatives. The monotonicity property (25) plausibly carries over to such a refined definition of $E x c l_{\mathrm{NC}}$. 
in terms of non-contradictoriness, thus illustrating concretely the equivalence between Blindness and Short-sightedness just established abstractly. For the present purpose, the challenge can be recalled as follows. In order to account for the oddness of sentence (27a), we need the mismatching implicature that Not all Italians come from a warm country. In order to get that implicature, we need to assume that the strengthened meaning (as well as overt only) is blind to the piece of common knowledge (26a).

(26) The actual common knowledge $c_{\text {actual }}$ entails:

a. That all Italians come from the same country.

b. That college presupposes high school.

a. \#Some Italians come from a warm country.

$=\varphi_{\text {some }}$

b. Mary has a college degree.

$=\varphi_{\text {college }}$

But why shouldn't then the strengthened meaning of sentence (27b) be blind to the piece of common knowledge (26b)? That would generate the mismatching implicature that Mary does not have a high school degree, thus incorrectly predicting sentence (26b) to sound odd as well (because it contradicts common knowledge on college admittance requirements).

Short-sightedness has an existential quantification over weakenings c of the actual common knowledge $\mathbf{c}_{\text {actual }}$ which makes it flexible enough to ignore either piece of common knowledge (26a) or (26b) thus failing to account for the oddness asymmetry between (27a) and (27b). Of course, it is not unconceivable that the two pieces of common knowledge (26a) and (26b) are not on equal footing. It might indeed be possible to provide independent evidence that the piece of common knowledge (26a) that Italians come form the same country is less "entrenched" or more "defeasible" than the piece of common knowledge (26b) that college presupposes high school. In that case, the unrestricted existential quantification over weakenings c which figures in the current formulation of Schlenker's Short-sightedness could be properly restricted in such a way to derive the asymmetry in (27). But as it stands, Short-sightedness has no advantage over Blindness. ${ }^{8}$

8 Short-sightedness does not help neither with Schlenker's original formulation of the challenge of contextually ordered alternatives, recalled in subsection 3.1. In fact, the two alternatives $\varphi_{\text {high school }}$ and $\varphi_{\text {college }}$ in (14) are contextually equivalent relative to Schlenker's special context (13). Allowing the oddness filter more context sensitivity thus does not help explaining why only $\varphi_{\text {high school }}$ but not $\varphi_{\text {college }}$ sounds odd in that context. 


\subsubsection{When exclusion is defined through asymmetric entailment}

Let me now turn to the case where the strengthened meaning used in the oddness filter is $[\llbracket \varphi]]]_{\mathrm{AE}}$, namely it is defined in terms of asymmetric entailment. Independently of oddness, this option is known to run into the following difficulty. In the proper context, sentence $\varphi_{\text {Adam }}$ in (28a) implies that Adam was the only student to solve the problem. In other words, $\varphi_{\text {Adam }}$ seems to trigger the implicature $\neg \psi_{\text {Bill }}$ that the alternative $\psi_{\text {Bill }}$ in (28b) is false. Yet, this alternative $\psi_{\text {Bill }}$ does not entail (let alone asymmetrically entail) the prejacent $\varphi_{\text {Adam }}$. Analogously, the sentence $\varphi_{\text {college }}$ in (29a) can trigger the implicature $\neg \psi_{\text {conservatory }}$ that the alternative $\psi_{\text {conservatory }}$ in (29b) is false; yet, this alternative $\psi_{\text {conservatory }}$ does not entail (let alone asymmetrically entail) the prejacent $\varphi_{\text {college }} .{ }^{9}$
a. Adam solved the problem.
$=\varphi_{\text {Adam }}$
b. Bill solved the problem.
$=\psi_{\text {Bill }}$
c. Adam solved the pbm and Bill solved the pbm.
$=\varphi_{\text {Bill }} \wedge \psi_{\text {Adam }}=\Sigma$
a. Adam has a college degree.
$=\varphi_{\text {college }}$
b. Adam has a conservatory degree.
$=\psi_{\text {conservatory }}$
c. Adam has college and conservatory degrees. $=\varphi_{\text {college }} \wedge \psi_{\text {conservatory }}=\Sigma$

Assume that the strengthened meaning and overt only share the same definition of excluded alternatives, as suggested in subsection 2.4. Then, the variants with overt only, namely Only Adam solved the problem and Adam only has a college degree, exacerbate this difficulty for a definition of excluded alternatives based on asymmetric entailment.

The only way out seems the following. The intended implicature of a sentence such as $\varphi_{\text {Adam }}$ in (28a) is derived not through the alternative $\psi_{\text {Bill }}$ in (28b), which fails the asymmetric entailment condition. Rather, it is derived through a different alternative $\Sigma$ which happens to entail both the prejacent $\varphi_{\text {Adam }}$ (thus displaying the required entailment) as well as the original alternative $\psi_{\text {Bill }}$ (thus providing the intended implicature). The simplest choice for this alternative $\Sigma$ is of course the conjunction of $\varphi_{\text {Adam }}$ and $\psi_{\text {Bill }}$, as in (28c). Analogous considerations lead to the postulation of the conjoined alternative $\Sigma$ in $(29 \mathrm{c})$. I conclude that the definition of excludability in terms of asymmetric entailment requires the assumption that, if $\psi$ is an alternative of a sentence $\varphi$, the corresponding conjoined alternative $\Sigma=\varphi \wedge \psi$ is an alternative of $\varphi$ as well.

Under the latter apparently unavoidable assumption on the set of scalar alternatives, Short-sightedness and Blindness turn out to be equivalent also when excludability is defined in terms of asymmetric entailment, as stated in (30).

9 Here, I have in mind the European school system, where music conservatory is not college and the two degrees can be pursued independently of each other. 


$$
\begin{array}{|lll|}
\hline \text { Blindeness: } & \text { Short-sightedness: } \\
\llbracket[\varphi \rrbracket]_{\mathrm{AE}}^{\mathrm{c}} \cap \mathrm{c}_{\text {actual }}=\emptyset & \Longleftrightarrow \begin{array}{l}
\llbracket[\varphi \rrbracket]_{\mathrm{AE}}^{\mathrm{c}} \cap \mathrm{c}_{\text {actual }}=\emptyset \\
\text { with } \mathrm{c}=\mathscr{W}
\end{array} \quad \text { for some } \mathrm{c} \supseteq \mathrm{c}_{\text {actual }} \\
\hline
\end{array}
$$

Again, the implication $\Longrightarrow$ trivially follows from the fact that $\mathrm{c}$ is existentially quantified over by Short-sightedness and that it is only subjected to the condition $\mathbf{c} \supseteq$ $\mathbf{c}_{\text {actual }}$, which is obviously satisfied by the position $\mathbf{c}=\mathscr{W}$ considered by Blindness. The reverse implication $\Longleftarrow$ follows from the obvious property (31) of the set $E x c l_{\mathrm{AE}}^{\mathrm{c}}$ of excluded alternatives defined in terms of asymmetric entailment: it is monotone relative to the common knowledge $\mathrm{c}$ when we restrict ourselves to the relevant alternatives, namely those obtained through conjunction with the prejacent.

(31) For any two contexts $c^{\prime}$ and $c^{\prime \prime}$ and any conjoined alternative $\Sigma=\varphi \wedge \psi$ :

$$
\text { If: } \mathbf{c}^{\prime} \subseteq \mathbf{c}^{\prime \prime}
$$

Then: $\quad \Sigma \in \operatorname{Excl}_{\mathrm{AE}}^{\mathrm{c}^{\prime}}(\varphi)$ entails $\Sigma \in \operatorname{Excl}_{\mathrm{AE}}^{\mathrm{c}^{\prime \prime}}(\varphi)$

It follows that any alternative $\Sigma=\varphi \wedge \psi$ negated by the short-sighted strengthened meaning $[\llbracket \varphi\rceil]]_{\mathrm{AE}}^{\mathrm{c}}$ is also excluded by the blind strengthened meaning $\left.\llbracket[\varphi]\right]_{\mathrm{AE}}^{\mathrm{c}=\mathscr{W}}$. If the former strengthened meaning is a contextual contradiction, the latter is a contextual contradiction as well. The equivalence in (30) is therefore established.

Let me take stock. Once excluded alternatives are defined in terms of asymmetric entailment, the set of alternatives of $\varphi$ needs to be enriched with these conjoined alternatives of the form $\Sigma=\varphi \wedge \psi$. This move effectively wipes out any difference between the two definitions based on asymmetric entailment and non-contradictoriness. As a consequence, the set of excluded alternatives displays analogous monotonicity properties (25) and (31), independently of whether it is defined in terms of asymmetric entailment or non-contradictoriness. In conclusion, Blindness and Short-sightedness turn out to be equivalent also when the strengthened meaning used in the oddness filter is $\|[\varphi \rrbracket]_{\mathrm{AE}}$, namely it is defined in terms of asymmetric entailment.

As reviewed in section 3, contextually ordered alternatives are problematic for Blindness. Let me show that Short-sightedness fares no better when the alternatives are defined in terms of asymmetric entailment, thus illustrating concretely the equivalence between Blindness and Short-sightedness just established abstractly. For the present purpose, the challenge raised by contextually ordered alternatives can be recalled as follows: in the proper context, the sentence $\varphi_{\text {college }}=$ Mary has a college degree triggers the scalar implicature that Mary lacks a conservatory degree but does not trigger the implicature that Mary lacks a high school degree, as $\varphi_{\text {college }}$ would otherwise sound odd because of a mismatch with the piece of common knowledge that college presupposes high school. These facts extend to the corresponding sentence with overt only. As we have just seen, an approach 
to scalar implicatures (or overt only) committed to asymmetric entailment needs to allow for conjoined alternatives such as those in (32). For instance, the conjoined alternative $\varphi_{\text {college }} \wedge \psi_{\text {conservatory }}$ in (32a) is needed in order for our sentence $\varphi_{\text {college }}$ to trigger the implicature that Mary lacks a conservatory degree.

a. Mary has college and conservatory degrees. $=\varphi_{\text {college }} \wedge \psi_{\text {conservatory }}$

b. Mary has college and high school degrees. $\quad=\varphi_{\text {college }} \wedge \psi_{\text {high school }}$

Short-sightedness allows the actual common knowledge $\mathbf{c}_{\text {actual }}$ to be slightly weakened to a knowledge $\mathrm{c}$ which fails at entailing the proposition that college presupposes high school. The conjoined alternative $\varphi_{\text {college }} \wedge \psi_{\text {high school }}$ in (32b) asymmetrically entails $\varphi_{\text {college }}$ relative to this weakened common knowledge c. Hence,


alternatives excluded by the sentence $\varphi_{\text {college }}$ relative to c. The short-sighted oddness filter (just like the blind filter) thus incorrectly predicts the sentence $\varphi_{\text {college }}$ to be odd, because its short-sighted strengthened meaning $\left[\left[\left[\varphi_{\text {college }}\right]\right]\right]_{\mathrm{AE}}^{\mathrm{c}}$ entails in particular that $\varphi_{\text {college }} \wedge \neg\left(\varphi_{\text {college }} \wedge \psi_{\text {high school }}\right)$, namely that Mary has a college degree but no high school degree.

In order to rescue sentence $\varphi_{\text {college }}$ from oddness, it would be crucial to neutralize the conjoined alternative $\varphi_{\text {college }} \wedge \psi_{\text {high school }}$ in (32b) - although crucially not the conjoined alternative $\varphi_{\text {college }} \wedge \psi_{\text {conservatory }}$ in (32a), which is needed to get the implicature that Mary lacks a conservatory degree. A possible way to achieve that would be to exploit the fact that $\varphi_{\text {college }} \wedge \psi_{\text {high school }}$ is contextually equivalent to $\varphi_{\text {college }}$ relative to the actual common knowledge $\mathrm{c}_{\text {actual }}$ and yet "longer" and thus "worse". This would require advocating something like the following procedure:

Step 1: construct the set of basic (i.e., non-conjoined) relevant scalar alternatives.

Step 2: add the alternatives obtained through conjunction of a base alternatives with the prejacent.

Step 3: take the actual common knowledge $\mathrm{c}_{\text {actual }}$ into account and prune those conjoined alternatives which are equivalent to the prejacent relative to $\mathbf{c}_{\text {actual }}$.

Step 4: consider a weakening $\mathbf{c} \supseteq \mathbf{c}_{\text {actual }}$ of the actual common knowledge $\mathrm{c}_{\mathrm{actual}}$ and compute the strengthened meaning using asymmetric entailment relative to $\mathbf{c}$, not relative to $\mathbf{C}_{\mathrm{actual}}$

Note that in step 3 it is crucial that we only eliminate the contextually equivalent conjoined alternatives, not the contextually equivalent basic alternatives, as otherwise we lose the leverage to derive the oddness of basic cases such as Some Italians come 
from a warm country. The intuition indeed ought to be that conjoined alternatives are "worse" than basic alternatives and thus more prone to be eliminated (or never generated).

Yet, I submit that even this maneuvering would fail at protecting the sentence $\varphi_{\text {college }}$ from being incorrectly labeled as odd by the Short-sighted oddness filter. To illustrate, common knowledge about the Italian school system entails that there are two types of high school, one with a scientific orientation (liceo scientifico) and one with a focus on the humanities (liceo classico). Both provide access to college. The challenge raised by the single alternative $\varphi_{\text {college }} \wedge \psi_{\text {high school }}$ in (32b) can now be mimicked with the conjoined action of the two following alternatives (34).

a. Bill has college and liceo scientifico degrees. $=\varphi_{\text {college }} \wedge \psi_{\text {liceo scientifico }}$

b. Bill has college and liceo classico degrees. $\quad=\varphi_{\text {college }} \wedge \psi_{\text {liceo classico }}$

Common knowledge has nothing to say concerning Mary's inclination for the hard sciences or the humanities. Hence, the two alternatives $\varphi_{\text {college }} \wedge \psi_{\text {liceo scientifico }}$ and $\varphi_{\text {college }} \wedge \psi_{\text {liceo classico }}$ are not ruled out at step 3 of the procedure (33), because they are not contextually equivalent to the base sentence $\varphi_{\text {college }}$.

\section{Gricean credentials}

Blindness (10) and Short-sightedness (23) both assume that the common knowledge c considered in the strengthened meaning $[[[\varphi]]]^{\mathbf{c}}$ evaluated by the oddness filter is different from the actual knowledge $\mathbf{c}_{\text {actual }}$. How can this assumption be made sense of? Subsection 2.4 has tackled this question for Blindness. This section reviews and challenges Schlenker's pragmatic answer to this question for Short-sightedness.

\subsection{Fallibility}

Schlenker suggests that Short-sightedness can be made to follow from Gricean reasoning. His proposal has two ingredients. The first ingredient is (35), which Schlenker dubs Fallibility because it captures the intuition that "communicative agents are fallible, and that there is always a very slight chance that the addressee might forget information about the context set" (p. 404). The second ingredient is (36), which "adapt[s] standard Gricean principles of preference to a framework that countenances Fallibility".

(35) "At any point $t$ in a conversation, for any proposition $p$ which was believed by the addressee at $t-1$, there is a small chance that an error will make the addressee forget $p$ " (p. 405).

(36) "If a sentence $S$ transmits to the addressee at least as much true information as sentence $S^{\prime}$ in all cases, and transmits strictly more true information than 
$S^{\prime}$ in some cases triggered by Fallibility, then it is to be preferred to $S^{\prime \prime}$, (p. 406).

To illustrate, the sentence $S=($ All) Italians come from a warm country makes a better contribution to the pragmatics of the conversation than the alternative $S^{\prime}=$ Some Italians come from a warm country, whereby the oddness of the latter. In fact, $S$ is contextually equivalent to $S^{\prime}$, whereby it transmits at least as much true information in all cases (where Fallibility does not apply). Furthermore, Fallibility requires the speaker to entertain the possibility that the addressee is momentarily oblivious to the fact that Italians all come from Italy. In such a circumstance, the universally quantified sentence $S$ transmits strictly more true information than the existentially quantified sentence $S^{\prime}$.

\subsection{Griceanizability}

Is it plausible to assume that the pragmatics of the conversation requires the speaker to entertain the hypothesis that the fallible addressee might have blanked out the fact that Italians come from Italy? Having lived in France for a few years, I understand that this assumption of a fallible addressee might capture the proverbial arrogance of the French speaker. Yet, outside of French pragmatics, Fallibility seems to me to be of little help. Indeed, let me say that the computation of scalar implicatures is griceanizable provided the mapping $\varphi \mapsto \llbracket[\varphi \rrbracket]]^{\mathrm{c}}$ which associates (the plain meaning of) an utterance with its strengthened meaning can be implemented through an algorithm whose steps all admit an independent motivation rooted in the phenomenology of human social interaction - and thus need not be encoded as idiosyncratic assumptions of a specialized grammatical module. Because of this focus on independent pragmatic motivations, establishing griceanizability is a task which pertains more to social psychology than to linguistics (anecdotical evidence from recommendation letters belongs indeed to folk social psychology). Does Fallibility contribute to the griceanizability of the algorithm for the computation of scalar implicatures? The answer really depends on whether it admits the required independent pragmatic justification. Currently, it does not: "fallibility is a technical assumption which is largely justified theory-internally", as Schlenker admits (p. 407). I conclude that, as it stands, Fallibility contributes nothing to the Griceanizability of Short-sightedness. It is nothing more than a reformulation of the sheer stipulation it was meant to derive, namely that the strengthened meaning evaluated by the oddness filter is not computed relative to the actual common knowledge. 


\section{A closer look at the challenge of contextually ordered alternatives}

Hirschberg's contextually ordered alternatives raise a challenge for contextual Blindness, as seen in section 3. Schlenker therefore suggests to replace Blindness with a more moderate contextual Short-sightedness. Yet, Blindness and Short-sightedness turn out to be equivalent, as seen in section 4. It thus looks like we are left with no ammunitions against the challenge of contextually ordered alternatives. ${ }^{10}$ This section thus takes a closer look at the challenge. The goal is to provide some initial evidence in favor of the following conjecture. Whenever contextual ordering seems to drive a certain pattern of scalar behavior, there is actually more logical structure than meets the eye and provides a logical ordering homorphous to the contextual ordering. It is the logical ordering (not the contextual ordering) which drives the scalar behavior. The contextual ordering thus never plays any role in scalar behavior, as predicted by Blindness (or the equivalent Short-sightedness). This section admittedly offers only a preliminary and tentative discussion of this conjecture, deferring its thorough investigation to future work.

\subsection{Alleged contextually ordered alternatives require specific lexical choices}

Let me say that two alternatives $\varphi, \psi$ form a Hirschberg (contextually ordered) pair $\langle\varphi, \psi\rangle$ provided they satisfy the three conditions (37).
a. $\varphi$ and $\psi$ are logically independent: neither logically entails the other.
b. $\psi$ asymmetrically entails $\varphi$ relative to the actual common knowledge.
c. 'only $\varphi$ ' and $\llbracket[\varphi \rrbracket]$ entail $\neg \psi$; but 'only $\psi$ ' and $\llbracket[\psi \rrbracket]$ do not entail $\neg \varphi$.

10 Danny Fox (p.c.) pointed out to me a solution to this impasse based on relevance. Here is the idea. Consider again the basic case of the odd sentence $\varphi_{\text {some }}=$ Some Italians come from a warm country. Assume that the algorithm for scalar implicatures is indeed blind to common knowledge. The algorithm will thus generate the implicature that $\psi_{\text {all }}=$ All Italians come from a warm country is false. But why is that implicature locked in place (condemning the sentence to oddness) rather than automatically cancelled (say, because of the contextual contradiction)? In Magri (2011), I propose the following answer: the mismatching implicature is mandatory because $\psi_{\text {all }}$ is necessarily relevant due the the fact that $\varphi_{\text {some }}$ is relevant (because it has been uttered), that $\varphi_{\text {some }}, \psi_{\text {all }}$ are contextually equivalent, and that relevance is closed relative to contextual equivalence. Fox notes that the sentence $\varphi_{\text {college }}=$ Mary has a college degree is not contextually equivalent to the alternative $\psi_{\text {high school }}=$ Mary has a high school degree in the out-of-the-blue context considered in subsection 3.2. Therefore, the inference $\neg \psi_{\text {high school }}$ is not a mandatory implicature of $\varphi_{\text {college }}$. In other words, the theory of oddness based on mismatching scalar implicatures does not strictly speaking predict $\varphi_{\text {college }}$ to be odd, as desired. Unfortunately, Fox's suggestion does not extend to Schlenker's special context considered in subsection 3.1, as the two alternatives $\varphi_{\text {college }}, \psi_{\text {high school }}$ are indeed contextually equivalent in that case. Furthermore, it does not extend to contrasts such as (39) and (41) discussed below in this section. 
To illustrate, the two alternatives in (38) are a Hirschberg pair. In fact, they are not logically ordered, as prescribed by (37a). Yet, they are ordered relative to the piece of common knowledge that college presupposes high school, as prescribed by (37b). Finally, they display the asymmetric behavior prescribed by (37c). In fact, sentence $\varphi=$ Mary has a high school degree implies (and the variant with overt only entails) that $\neg \psi$, namely that Mary dropped out of school after high school and thus did not attend college; but sentence $\psi=$ Mary has a college degree does not imply (nor does the variant with overt only say) that $\neg \varphi$, namely that Mary skipped high school.

$$
\langle\varphi=\text { Mary has a high school degree, } \psi=\text { Mary has a college degree }\rangle
$$

Hirschberg alternatives display a behavior (37c) which mirrors their contextual ordering (37b), not their (lack of) logical ordering (37a). If scalar implicatures and overt only were contextually blind (or short-sighted), Hirschberg pairs should not exist. This is the essence of the challenge reviewed in section 3.

I would like to take the edge off this challenge as follows. If scalar implicatures and overt only were indeed sensitive to the actual common knowledge, it should be very easy to construct Hirschberg pairs. Indeed, Hirschberg (1991: p. 83) herself seems to suggest that any two logically independent alternatives could be turned into a Hirschberg pair through the proper manipulation of the common knowledge: "I will propose a new characterization of these orderings [that support scalar implicatures] as partially [contextually] ordered sets and claim that any poset can support scalar implicatures". Instead, I submit that Hirschberg pairs are restricted and crucially dependent on specific lexical choices. To start, let me point out that a number of examples considered in Hirschberg (1991: chapter 8) (building on earlier literature, such as Harnish 1979; Horn 1972; Kempson 1975; Gazdar 1979, 1980; Prince 1982; Walker 1975; Levinson 1983, etcetera) do not actually qualify as Hirschberg pairs to start with. For instance, she discusses scales such as $\langle$ acquaintance, friend, lover $\rangle$ and $\langle$ private, corporal, sergeant $\rangle$ and remarks (p. 98) that "while lovers may also be friends, they need not be-and sergeants cannot be privates", thus concluding that the corresponding alternatives are not ordered by any entailment. Furthermore, they do not display the asymmetric behavior required by (37c): the sentence John is only a friend implies that he is not a lover and the sentence John is (only) a lover can be read as implying that he is not also a friend.

Sentence (39a) is instead a pertinent example. It is fine in the context considered and it says that the train did not make it to stop $D$ but it does not imply that the train skipped any of the stops preceding $C$. This example indeed illustrates a class of examples discussed in Hirschberg (1991: subsection 5.1.8). Since the ordering of the stops obviously pertains to the common knowledge, the two alternatives in (40a) count as a Hirschberg pair.

(39) Context: I go to work by train. The train route is $A \rightarrow B \rightarrow C \rightarrow D \rightarrow E$. 
The train always leaves from station $A$, and goes through one station at the time. Sometimes though, the train breaks down before it gets to the final station $E$.

a. Yesterday, the train only arrived at station $C$.

b. \#Yesterday, the train only stopped/made a stop at station $C$.

a. $\langle\varphi=$ the train arrived at $\mathrm{B}, \psi=$ the train arrived at $\mathrm{C}\rangle$

b. $\langle\varphi=$ the train stopped at $\mathrm{B}, \psi=$ the train stopped at $\mathrm{C}\rangle$

Crucially, sentence (39b) instead sounds odd in the same context, although it only minimally differs for the choice of the main predicate. Intuitively, oddness is due to the fact that (39b) implies that the train skipped the stops preceding $C$. In other words, when only is applied to any of the two alternatives in (40b), it excludes the other, despite the fact that the two alternatives are contextually ordered. The two alternatives in (40b) are therefore not a Hirschberg pair, because their symmetric behavior does not mirror their contextual asymmetric entailment relationship. ${ }^{11}$ This contrast seems to me surprising under the assumption that the semantics of overt only as well as the computation of scalar implicatures are sensitive to contextual information. Under this assumption, they should be able to detect the contextual asymmetric entailment between the two alternatives in both pairs (40a) and (40b) and thus yield a fine upper-bounded meaning in both cases (39a) and (39b).

Examples like this can be easily multiplied. Another analogous contrast is provided in (41). Sentence (41a) is fine in the context considered and thus does not imply that Mary skipped introductory Calculus. The two alternatives in (42a) thus count as a Hirschberg pair. But sentence (41b), which only differs because the main predicate has been changed to took, is odd because it implies that Mary skipped introductory Calculus. The two alternatives in (42b) therefore do not form a Hirschberg pair.

11 In (39) and in the rest of this section, I consider sentences containing overt only, which makes the judgments sharper. I submit nonetheless that the relevant judgments extend to the corresponding sentences without overt only. For instance, the contrast in (39) does extend to the corresponding sentences without only, at least when they are construed as answers to a proper question, such as Where did the train arrive/stop yesterday? Furthermore, there is indeed a contrast between sentences (ia) and (ib) relative to the actual world where the train stoppes are lined up as specified.

(i) a. \#Yesterday, the train arrived at A, B and C.

b. Yesterday, the train stopped at A, B and C.

It is tempting to explain this contrast as follows. Sentence (ia) is deviant because it is equivalent to the shorter sentence The train arrived at $C$. Sentence (ib) is instead fine because it is not equivalent to the shorter sentence The train stopped at $C$, as the latter does trigger the inference that the train did not stop at $A$ and $B$. 
(41) Context: Prof. Smith teaches introductory Calculus, intermediate Calculus, and advanced Calculus. He is strict about requirements and never allows any student to take one of his Calculus classes without having taken the preceding one(s). While in college, did Mary take any classes with him?

a. Mary only got to intermediate Calculus.

b. \#Mary only took intermediate Calculus.

a. $\langle\varphi=$ Mary got to introductory Calculus, $\psi=$ Mary got to intermediate Calculus $\rangle$

b. $\langle\varphi=$ Mary took introductory Calculus, $\psi=$ Mary took intermediate Calculus $\rangle$

If only and scalar implicatures were sensitive to contextual ordering, why should there be any difference between the pairs of alternatives (42a) and (42b)?

Let me consider again the very same example about school degrees used in section 3.2 to challenge Blindness. As a reminder, the crucial observation is that sentence (43a) says that Mary lacks a college degree but it says nothing about her junior high school degree. In other words, the two alternatives (38), repeated in (44a), count as a Hirschberg pair. Sentence (43b) instead cannot be construed as saying that Mary has transcripts only up to high school (because she only attended up to high school and then skipped college). In other words, the two alternatives (44b) do not count as a Hirschberg pair.

a. Mary only has a high school degree.

b. Mary only has transcripts from high school.

c. Maria ha frequentato solo il liceo. Mary has frequented only the high school

(44) a. $\langle\varphi=$ Mary has a junior high degree, $\psi=$ Mary has a high school degree $\rangle$

b. $\langle\varphi=$ Mary has junior high school transcripts, $\psi=$ Mary has high school transcripts $\rangle$

c. $\langle\varphi=$ Maria ha frequentato le scuole medie, $\psi=$ Maria ha frequentato il liceo $\rangle$

Furthermore, Italian has a predicate frequentare (same as French fréquenter) which roughly means "sit through" and thus loosely translates English attend. Despite sentence (43c) providing a close translation of the original sentence (43a), it does seem to me to imply that Maria skipped elementary and junior high school (as well as that she did not get to college). ${ }^{12}$ In other words, the two alternatives (44c) do not count as a Hirschberg pair neither. Crucially, the original sentence (43a) and the variants (43b) and (43c) tap into the same piece of common knowledge concerning the school system. How could a context sensitive only (and a context sensitive

12 Two French informants and two Italian informants confirmed this intuition; the judgment for the English sentence with the predicate attend seems less clear to me. 
strengthened meaning) be sensitive to that common knowledge only in the former case?

Let me close with the observation that not only overt only and scalar implicatures seem to be sensitive to this subtle distinction between Hirschberg and non-Hirschberg pairs. Also the acceptability of which- $N_{\text {sing }}$ questions where $N_{\text {sing }}$ is a singular noun seems to make the same distinction. Indeed, Hirschberg pairs yield the felicitous which- $N_{\text {sing }}$ questions in (45): these questions do not imply that the train made a unique stop, that the addressee took a unique Calculus class, or that (s)he has a unique school degree. Non-Hirschberg pairs instead yield the questions in (46) which are infelicitous precisely because they trigger these undesired implications.

a. Which stop did the train arrive to?

b. Which level of Calculus did you get to?

c. Which school degree do you have?

a. Which stop did the train stop at?

b. Which Calculus class did you take?

c. Which school do you have transcripts of?

d. Quale scuola hai frequentato? which school have-you attended?

Let me take stock. Hirschberg pairs such as (40a), (42a), and (44a) come with minimal variants (40b), (42b), and (44b)-(44c) which are not Hirschberg pairs. In other words, Hirschberg pairs require specific lexical choices: arrive at stop $C$ instead of stop at $C$; get to intermediate Calculus instead of take intermediate Calculus; attend high school instead of frequentare il liceo. If scalar implicatures and overt only had access to contextual ordering, why should these minimal lexical differences matter? The data presented in this subsection thus take the edge off the challenge against Blindness (or the equivalent Short-sightedness) discussed in section 3 .

\subsection{Alleged contextually ordered alternatives involve logical ordering}

The challenge nonetheless remains: how can Blindness cope with Hirschberg pairs such as (40a), (42a), and (44a), where it looks like contextual ordering is driving the scalar behavior? ${ }^{13}$ This subsection offers a tentative answer to this question,

13 The complementary cases (40b), (42b), and (44b)-(44c) which do not yield Hirschberg pairs follow straightforwardly from the assumption that the set of excluded alternatives which appears in the definition (4) of the strengthened meaning and the semantics (12) of overt only is blind to contextual information and defined in terms of non-contradictoriness as in (6). Since the two alternatives in (40b) and those in (42b) are logically unordered, the symmetric scalar behavior displayed by these non-Hirschberg pairs follows straightforwardly. 
although I acknowledge that the range of facts considered in the preceding subsection is admittedly too restricted to warrant any solid conclusions. I would like to put forward the conjecture that in these cases problematic for Blindness there is actually a logical ordering which happens to be homomorphous to the contextual ordering. It is not the contextual ordering but the logical ordering which is driving the scalar behavior, as predicted by Blindness (or the equivalent Short-sightedness). I will explore two instances of this logical ordering: the ordering among degrees $\left(d^{\prime} \gtrless d\right)$ and the ordering among events $\left(e^{\prime} \supsetneq e\right)$.

\subsubsection{Logical ordering of degrees: $d^{\prime} \gtrless d$}

Let me start with the original case discussed in subsection 3.2, repeated once more in (47). The challenge is to account for the fact that the sentence $\varphi_{\text {high school }}$ implies that Mary lacks a college degree while the sentence $\varphi_{\text {college }}$ does not imply that Mary lacks a high school degree.
a. $\varphi_{\text {high school }}=$ Mary has a high school degree
b. $\varphi_{\text {college }}=$ Mary has a college degree
$\begin{aligned} \rightsquigarrow & \rightsquigarrow \varphi_{\text {college }} \\ \not \varphi & \end{aligned}$

Schlenker interprets this asymmetry (47) as evidence that the strengthened meaning of $\varphi_{\text {high school }}$ is $\varphi_{\text {high school }} \wedge \neg \varphi_{\text {college }}$ but that the strengthened meaning of $\varphi_{\text {college }}$ is not $\varphi_{\text {college }} \wedge \neg \varphi_{\text {high school }}$. I would like to explore a different approach. Assume that high school degree is actually a degree expression, just like the numeral two. It is only a matter of common knowledge that the degree denoted by one is smaller than the degree denoted by two. Or that the expression high school degree denotes a degree of education which is smaller than the degree denoted by college degree. Since scalar implicatures and overt only are blind to common knowledge, they are in particular blind to this information. Yet, the ordering of degrees (together with the monotonicity of the environment where the degree expression occurs) suffices for Blindness to only exclude the alternatives corresponding to larger degrees, without also negating the alternatives corresponding to smaller degrees. In other words, the strengthened meaning of the sentence Mary has a high school degree (or equivalently the plain meaning of the corresponding sentence with overt only) is (48a). This pragma-semantics is completely analogous to the one in (48b) for the sentence Mary has two children with the degree expression two.

a. has-education $\left(m, d_{\text {high school }}\right) \wedge \bigwedge_{d \gtrless d_{\text {high school }}} \neg$ has-education $(m, d)$

b. has-children $(m, 2) \wedge \bigwedge_{d \geq 2} \neg$ has-children $(m, d)$

The predicate has-education in (48a) holds of Mary and a degree $d$ if and only if Mary has a level of education which is equal to or higher than the degree of education 
$d$. The strengthened meaning (48a) excludes has-education $(m, d)$ for any degree $d$ larger than the degree of education $d_{\text {high school }}$ denoted by high school degree. The computation of this strengthened meaning requires no mundane knowledge about high schools, colleges and their admittance requirements. Consider the variants of the alternatives (47) obtained by replacing has a degree with has transcripts and with the Italian frequentare. As noted above, the alternatives (44b) and (44c) thus obtained do not count as Hirschberg pairs. This would now follow easily from the plausible assumption that the alternatives thus obtained cannot be construed as degree expressions.

As recalled in subsection 3.1, Schlenker adds an interesting twist to the asymmetry in (47): he points out that this asymmetry persists even when common knowledge is tampered with to the effect of replacing the out-of-the-blue contextual asymmetric entailment between $\varphi_{\text {college }}$ and $\varphi_{\text {high school }}$ with contextual equivalence, as in his scenario (13), where every employee has a high school degree if and only if (s)he has a college degree. As I argued above, Schlenker's observation (far from providing an argument against Blindness) suggests that contextual entailment is plausibly not playing any role in the proper derivation of the asymmetric behavior in (47), as the asymmetry persists even without contextual ordering. The proposal sketched here, that the asymmetry (47) follows from logical properties of the ordering of degrees of education through the pragma-semantics in (48), instead makes sense of Schlenker's observation: tampering with the common knowledge should have no effect on the asymmetry.

\subsubsection{Logical ordering of events: $e^{\prime} \supsetneq e$}

I will now extend the preceding account to the other cases discussed in subsection 6.1 , only using the mereological ordering of events instead of the ordering of degrees. To start, consider the observation (39) that arrive yields a Hirschberg pair while stop does not. What could be the relevant difference? I submit the following intuition. In

the actual world, the event $e_{B}^{\text {stop }}$ of the train stopping at $B$ and the event $e_{C}^{\text {stop }}$ of the train stopping at $C$ are disjoint, independent events, as depicted in (49a).

a.

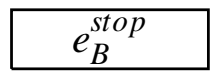

b.

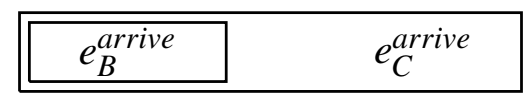

The case of arriving events is different. I submit that in the actual world, the event $e_{B}^{\text {arrive }}$ of the train arriving at $B$ is a subset of the event $e_{C}^{\text {arrive }}$ of the train arriving 
at $C$, as depicted in (49b). ${ }^{14}$ In other words, what is special about the Hirschberg pair of alternatives The train arrived at $B$ and The train arrived at $C$ is that the contextual ordering of the alternatives is matched by the mereological ordering of the corresponding events.

Analogous considerations hold for the case in (41), whereby the predicate got to introductory/intermediate Calculus yields a Hirschberg pair while the predicate took introductory/intermediate Calculus does not. I submit that in the actual world, the event $e_{\text {intro }}^{\text {take }}$ of Mary taking introductory Calculus and the event $e_{\text {inter }}^{\text {take }}$ of Mary taking intermediate Calculus are disjoint, independent events, as represented in (50a).

a.

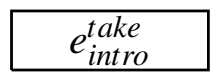

b.

\begin{tabular}{|l|l|}
\hline$e_{\text {intro }}^{\text {get }}$ & $e_{\text {inter }}^{\text {get }}$ \\
\hline
\end{tabular}

The case of get to is different. I submit that in the actual world, the event $e_{\text {intro }}^{\text {get }}$ of Mary getting to introductory Calculus is a subset of the event $e_{\text {inter }}^{\text {get }}$ of Mary getting to intermediate Calculus, as depicted in (50b). In other words, what is special about the Hirschberg pair of alternatives Mary got to introductory Calculus and Mary got to intermediate Calculus is that the contextual ordering of the alternatives is matched by the mereological ordering of the corresponding events.

All cases considered so far which fail at yielding a Hirschberg pair can be rescued by adding up to, as shown by the contrasts in (51)-(54).

a. \#Yesterday, the train only stopped/made a stop at station $C$.

14 Recent advances in the semantics of telic motion predicates support the conjecture that arriving events have the mereological structure depicted in (49b), along the following lines. Building on a large literature (which includes Krifka 1998; Hay, Kennedy \& Levin 1999; Kennedy \& Levin 2008; Beavers 2008), Beavers (2013: section 2) proposes the scalar semantics (i) for a sentence such as John walked to the station with the telic motion predicate walk. Here, $s$ is a variable over path scales. The predicate walk is a three-way relation between an event, a theme, and a path scale, as represented by the first conjunct in (i). This relation encodes the fact that $j$ is the agent of a walking event $e$ along the path scale $s$. The event and scale arguments are connected through a proper homomorphism between the sub-events of $e$ and the points of the path scale $s$ (e.g., temporal adjacency in $e$ corresponds to degree adjacency in $s$ ). The other conjunct in (i) captures the condition that the station is the goal or the end point of the walking event $e$ relative to the path scale $s$.

(i) $\exists e \exists s[\mathbf{w a l k}(e, s, \mathbf{j o h n}) \wedge \operatorname{GOAL}(e, s$, the station $)]$

This semantics plausibly extends from walk to to arrive at. The assumption that $e_{B}$ is an event of the train arriving at $B$ thus means that there exists a path scale $s_{B}$ such that $\operatorname{arrive}\left(e_{B}, s_{B}\right.$, train). Analogously, the assumption that $e_{C}$ is an event of the train arriving at $C$ means that there exists a path scale $s_{C}$ such that arrive $\left(e_{C}, s_{C}\right.$, train). The linear ordering of the stops along the railroad entails that $s_{B} \subseteq s_{C}$. It is not implausible that the homomorphism between events and path scales ensures that $s_{B} \subseteq s_{C}$ entails $e_{B} \subseteq e_{C}$, yielding the mereological structure depicted in (49b). 
b. Yesterday, the train only stopped/made a stop up to station $C$.

a. \#Mary (only) took intermediate Calculus.

b. Mary (only) took up to intermediate Calculus.

a. \#Mary (only) has transcripts for high school.

b. Mary (only) only has transcripts up to high school.

a. \#Maria ha frequentato solo il liceo.

Mary has attended only high school

b. Maria ha frequentato solo fino al liceo.

Mary has attended only up to high school

Consider for instance the case in (51). I submit that up to turns the mereological structure (49a) of stopping events into the one in (55): in the actual world, the event $e_{B}^{\text {stopupto }}$ of the train stopping up to $B$ is a subset of the event $e_{C}^{\text {stopupto }}$ of the train stopping up to $C$. The operator up to thus turns the mereological structure of stopping events into that of arriving events.

\begin{tabular}{|c|c|}
\hline$e_{B}^{\text {stop upto }}$ & $e_{C}^{\text {stop upto }}$ \\
\hline
\end{tabular}

In other words, what is special about the Hirschberg pair of alternatives with up to in (51b)-(54b) is that the contextual ordering of the alternatives is matched by the mereological ordering of the corresponding events.

The evidence just reviewed supports the generalization that two alternatives form a Hirschberg pair only when the mereological structure of the corresponding events matches the contextual ordering of the two alternatives. Let me now show how this generalization can be used to account for the behavior of Hirschberg pairs despite Blindness. For concreteness, I focus on the contrast (39) between arrive and stop-analogous considerations hold for the other cases considered so far. Let me assume that the strengthened meaning of the sentence The train stopped at $C$ (or equivalently the plain meaning of the corresponding sentence with overt only) is (56a). The prejacent says that $e$ is an event of the train stopping at the location $C$. The excluded alternatives say that $e$ is the largest event of the train arriving (namely that there exists no larger arriving event $e^{\prime}$ ).

$$
\begin{aligned}
& \text { a. } \exists e[\underbrace{[\operatorname{stop}(e, \mathbf{t}) \wedge \operatorname{LOC}(e)=\mathbf{C}]}_{\text {prejacent }} \wedge \bigwedge_{e^{\prime} \supsetneq e} \neg \underbrace{\left[\operatorname{stop}\left(e^{\prime}, \mathbf{t}\right)\right]}_{\text {alternative }}] \\
& \text { b. } \exists e[\underbrace{[\operatorname{arrive}(e, \mathbf{t}) \wedge \operatorname{GOAL}(e)=\mathbf{C}]}_{\text {prejacent }} \wedge \bigwedge_{e^{\prime} \supsetneq e} \neg \underbrace{\left[\operatorname{arrive}\left(e^{\prime}, \mathbf{t}\right)\right]}_{\text {alternative }}]
\end{aligned}
$$

Analogous formalism holds for the sentence The train arrived at $C$, as spelled out in (56b). The only difference is that the event and the stop $C$ are now related 
through a function GOAL which maps an arriving event to its end point or goal (here I am using the terminology of Beavers 2008, 2013). The two formulas in (56) effectively say that the largest event of the train stopping/arriving is an event of the train stopping/arriving at $\mathrm{C}$.

The two formulas in (56) share the general shape (4) of the strengthened meaning or equivalently the general shape (12) of the plain meaning of a sentence containing overt only. Nonetheless, a principled derivation of the pragma-semantics (56) admittedly needs to overcome two difficulties which I leave open at this stage. The first difficulty is that existential closure over the event argument $e$ of the prejacent has wide scope over both the prejacent and the negated alternatives in (56). This allows the exclusion of only those alternatives which correspond to an event $e^{\prime}$ such that $e^{\prime} \supsetneq e$. This condition is analogous to the condition $d^{\prime} \gtrless d$ used above for degrees and it will indeed play just the same crucial role in what follows. ${ }^{15}$ The second difficulty against a principled derivation of the pragma-semantics (56) is how to derive the restriction $e^{\prime} \supsetneq e$ on the excluded alternatives from a general notion of excluded alternatives such as those discussed in subsection 2.2.

These strengthened meanings (56a) and (56b) do not take into account the difference between the lexical predicates arrive and stop-exactly as the strengthened meaning of the sentence Some P come from a warm country does not take into account whether $P$ is Italians or friends of mine. Furthermore, the proposed strengthened meanings do not rely on mundane knowledge concerning the train schedule or the linear ordering of the five stops $A$ through $E$ in the actual world. They only rely on the relation of inclusion $e^{\prime} \supsetneq e$ which is defined on the domain of events. They are thus analogous to the strengthened meaning in (48a), which does not rely on mundane knowledge concerning high school and college admission requirements but only relies on the ordering relation defined on the domain of degrees. I thus submit that the pragma-semantics in (56) is compatible with Blindness (or the equivalent Short-sightedness).

Let me now argue that (56) suffices to derive the contrast in (39), whereby the sentence with arrive sounds fine in the context considered while the variant with stop sounds odd because it triggers the implicature that the train skipped stop $B$. Let me start with the latter fact. Thus, I need to show that, if the strengthened meaning (56a) is true in the actual world, then the train skipped stop $B$. Indeed, suppose

15 If this assumption of a wide scope operator $\exists e$ turns out to be untenable, I would need to make use of variants of (56) such as the equivalent formula in (i), which nonetheless is not as close to the general scheme $(4) /(12)$ for the strengthened meaning and the meaning of overt only.

(i) $\exists e[\operatorname{stop}(e, \mathbf{t}) \wedge \operatorname{LOC}(e)=\mathbf{C}] \wedge \bigwedge_{e^{\prime}} \neg\left[\operatorname{stop}\left(e^{\prime}, \mathbf{t}\right) \wedge \exists e^{\prime \prime}\left[e^{\prime \prime} \subsetneq e^{\prime} \wedge \operatorname{stop}\left(e^{\prime \prime}, \mathbf{t}\right) \wedge \operatorname{LOC}\left(e^{\prime \prime}\right)=\mathbf{C}\right]\right]$ 
by contradiction that is not the case, namely that (56a) is true in the actual world and that the train did stop at $B$. Since (56a) is true in the actual world, there exists an event $e_{C}$ of the train stopping at $C$. The hypothesis that the train stopped at $B$ means that there exists also an event $e_{B}$ of the train stopping at $B$. Because of events' mereological structure, there exists the event $e_{B} \oplus e_{C}$ which is the sum of those two events. Assume that this sum event of two stopping events counts as a stopping event itself. This would follow from Kratzer's (in progress) Cumulatively hypothesis applied to the event predicate stop. Since the two events $e_{B}$ and $e_{C}$ are disjoint independent events as depicted in (49a), the sum event $e_{B} \oplus e_{C}$ properly includes the event $e_{C}$, as stated in (57). This proper inclusion holds irrespectively of whether the stop $B$ is on the train's path to $C$ in the actual world.

$$
e_{B} \oplus e_{C} \supsetneq e_{C} \text { in the actual world. }
$$

By (57), the formula (56a) is false in the actual world because the alternative corresponding to the event $e^{\prime}=e_{B} \oplus e_{C}$ is true. This conclusion yields the desired contradiction. In other words, the strengthened meaning (56b) effectively requires the train not to have made any stop anywhere else than at $C$, neither at the stops preceding $C$ nor at the stops following $C$.

Let me now turn to the fact that the sentence (39a) with arrive sounds fine in the envisioned scenario, namely it does not trigger the implicature that the train did not arrive at $B$. Indeed, assume that arriving events have the mereological structure depicted in (49b), whereby the event $e_{B}$ of the train arriving at $B$ is a sub-event of the event $e_{C}$ of the train arriving at $C$ in the actual world, because of the linear ordering of the train's stops. The sum of two events $e_{B}$ and $e_{C}$ of the train arriving at $B$ and the train arriving at $C$ is an event $e_{B} \oplus e_{C}$ which is not larger than but rather coincides with the event $e_{C}$ of the train arriving at $C$, as stated in (58).

$$
e_{B} \oplus e_{C}=e_{C} \text { in the actual world. }
$$

Because of (58), the only way to construct an arriving event $e^{\prime}$ which properly contains the arriving event $e_{C}$ is for the train to have reached at least a little bit further than $C$. In conclusion, the strengthened meaning (56b) with arrive does not mismatch with common knowledge, because it simply denies that the train went any further than stop $C$.

Let me take stock. Sticking with Blindness, the strengthened meanings (56a) and (56b) for the two sentences with stop and arrive are formally identical. The asymmetry observed in (39) follows from the fact that, when these two formally identical strengthened meanings are deployed against common knowledge, the different mereological properties of stopping and arriving events kick in. Because of these different mereological properties (49a) and (49b), the logical relation of inclusion among events is homomorphous with the linear ordering of the stops in 
the actual world in the case of arriving events but not in the case of stopping events. This explains why in the case of arrive, but crucially not in the case of stop, the scalar behavior seems to access the common knowledge concerning the ordering of the stops, despite the fact that the scalar behavior is actually blind to that knowledge and only sensitive to the logical ordering $e^{\prime} \supsetneq e$ of events.

\section{Conclusions}

Three types of alternatives have been considered in the literature on scalar implicatures (and overt only). The alternatives (59a) and (59b) behave asymmetrically: the former excludes the latter but not vice versa. This asymmetric behavior plausibly reflects their logical asymmetric entailment relationship. The alternatives (60a) and (60b) behave symmetrically: either one of the two excludes the other. This symmetric behavior plausibly reflects their (logical and contextual) independence.

\section{Logically ordered alternatives:}

a. Mary (only) met Adam or Bill. $\rightsquigarrow \neg$ Mary met Adam and Bill

b. Mary (only) met Adam and Bill.

$$
\Varangle \rightarrow \neg \text { Mary met Adam or Bill }
$$

(60) Unordered alternatives:

a. Mary (only) met Adam.

$\rightsquigarrow \neg$ Mary met Bill

b. Mary (only) met Bill.

$\rightsquigarrow \neg$ Mary met Adam

(61) Contextually ordered alternatives:

a. Mary has a high school degree. $\quad \rightsquigarrow \neg$ Mary has a college degree

b. Mary has a college degree. $\quad \downarrow_{\rightarrow} \neg$ Mary has a high school degree

Hirschberg (1991) submits that there exists a third relevant case. The alternatives (61a) and (61b) behave asymmetrically: the former excludes the latter but not vice versa. Yet, the two alternatives are not logically ordered. It is only by virtue of common knowledge that a college degree entails a high school degree. The asymmetric behavior of these two alternatives thus seems to mirror their contextual asymmetric entailment relationship, not their logical independence.

Magri (2009b,a, 2011) submits that the computation of scalar implicatures (as well as the semantics of overt only) is blind to common knowledge. Schlenker (2012) objects that Hirschberg's contextually ordered alternatives (61) defy this assumption, as their asymmetric behavior seems to require access to their contextual asymmetric entailment relationship. Schlenker thus replaces contextual Blindness with a more moderate assumption which I have dubbed contextual Short-sightedness, as it allows implicatures to retain some contextual sensitivity, hopefully as much as needed to deal with the contextual ordering of the alternatives in (61). In this paper, I have shown that Schlenker's Short-sightedness turns out to be equivalent to Blindness. 
His proposal is therefore a technical mistake that provides no new ammunitions against the challenge raised by contextually ordered alternatives (61). I have then taken a closer look at the challenge. I have presented some initial evidence that cases such as (61) are restricted to specific lexical choices. And I have conjectured that these lexical choices share the property of yielding a logical ordering which is congruent with the contextual ordering. Although a thorough investigation of this conjecture is left to future work, the conjecture opens the possibility that it is not the contextual ordering but the logical ordering which is driving the asymmetric scalar behavior in cases such as (61), thus reconciling these cases with Blindness (or the equivalent Short-sightedness).

\section{References}

Abusch, Dorit \& Mats Rooth. 2004. Empty domain effects for presuppositional and non-presuppositional determiners. In Barbara Partee \& Hans Kamo (eds.), Context dependence in the analysis of linguistic meaning, Elsevier.

Beaver, David \& Brady Clark. 2008. Sense and sensitivity. Wiley-Blackwell.

Beavers, John. 2008. Scalar complexity and the structure of events. In Johannes D olling, Tatjana Heyde-Zybatow \& Martin Sch afer (eds.), Event structures in linguistic form and interpretation, 245-265. Mouton de Gruyter.

Beavers, John. 2013. Aspectual classes and scales of change. Linguistics 51. 681-706.

Chierchia, Gennaro. 2004. Scalar implicatures, polarity phenomena and the syntax/pragmatics interface. In Adriana Belletti (ed.), Structures and beyond, Oxford: Oxford University Press.

Chierchia, Gennaro, Danny Fox \& Benjamin Spector. 2012. The grammatical view of scalar implicatures and the relationship between semantics and pragmatics. In Paul Portner, Claudia Maienborn \& Klaus von Heusinger (eds.), Handbook of Semantics, vol. 3, Berlin, New York: Mouton de Gruyter.

Fox, Danny. 2000. Economy and semantic interpretation. MIT Press: Cambridge, Mass.

Fox, Danny. 2007. Free choice and the theory of scalar implicatures. In Uli Sauerland \& Penka Stateva (eds.), Presupposition and implicature in compositional semantics, 71-120. New York: Palgrave Macmillan.

Gazdar, Gerald. 1979. Pragmatics: Implicature, presupposition and logical form. New York: Academic Press.

Gazdar, Gerard. 1980. Pragmatics and logical form. Journal of Pragmatics 4. 1-13. Geurts, Bart. 2007. Existential import. In Ileana Comorovski \& Klaus von Heusinger (eds.), Existence: Semantics and syntax, 253-271. Springer.

Geurts, Bart. 2010. Quantity implicatures. Cambridge University Press. 
Harnish, Robert M. 1979. Logical form and implicature. In Kent Bach \& Robert M. Harnish (eds.), Linguistic communication and speech acts, 313-391. Cambridge, MA: MIT Press.

Hart, H. 1951. A logician's fairy tale. The philosophical review 60. 198-212.

Hay, Jennifer, Christopher Kennedy \& Beth Levin. 1999. Scalar structure underlies felicity in degree achievements. In Tanya Matthews \& Devon Strolovitch (eds.), Proceedings of Semantics and Linguistic Theory IX, 127-144. CLC Publications. Hirschberg, Julia. 1991. A theory of scalar implicature Outstanding Dissertations in Linguistics. Garland.

Horn, L. 1972. On the semantic properties of logical operators in English: UCLA dissertation. Distributed by IULC.

Horn, Larry. 1997. All john's children are as bald as the king of france: existential import and the geometry of opposition. In CLS 33, 155-179.

Horn, Laurence R. 2005. The border wars: a neo-gricean perspective. In Ken Turner \& Klaus von Heusinger (eds.), Where Semantics meets Pragmatics, Elsevir.

de Jong, Franciska \& Henk Verkuyl. 1991. Generalized quantifiers: the properness of their strength. In John van Benthem \& Alice ter Meulen (eds.), Generalized quantifiers in natural language, 21-43. Foris.

Kempson, Ruth M. 1975. Presupposition and the delimitation of semantics, vol. 15 Cambridge Studies in Linguistics. Cambridge, England: Cambridge University Press.

Kennedy, Christopher \& Beth Levin. 2008. Measure of change: the adjectival core of degree achievements. In Louise McNally \& Christopher Kennedy (eds.), Adjectives and adverbs: Syntax, semantics, and discourse, 156-182. Oxford University Press.

Kratzer, Angelika. in progress. The event argument and the semantics of verbs. Univ. of Massachusetts at Amherst; available online at www.semanticarchive.net.

Krifka, Manfred. 1998. The origins of telicity. In Susan Rothstein (ed.), Events and grammar, 197-235. Kluwer.

Lappin, Shalom \& Tanya Reinhart. 1988. Presuppositional effects of strong determiners: a processing account. Linguistics 26. 1021-1037.

Levinson, Stephen C. 1983. Pragmatics. Cambridge: Cambridge University Press.

Magri, Giorgio. 2009a. A theory of individual-level predicates based on blind mandatory scalar implicatures. Natural Language Semantics 17(3). 245-297. doi:10.1007/s11050-009-9042-x.

Magri, Giorgio. 2009b. A theory of individual-level predicates based on blind mandatory scalar implicatures. Constraint promotion for Optimality Theory: MIT dissertation.

Magri, Giorgio. 2011. Another argument for embedded scalar implicatures based on oddness in downward entailing contexts. Semantics \& Pragmatics 4. 1-51. 
doi:10.3765/sp.4.6.

Pistoia-Reda, Salvatore. to appear. Contextual blindness in implicature computation. Natural Language Semantics .

Prince, E. F. 1982. Grice and universality: a reappraisal. In Proceedings of the penn linguistics colloquium, .

Russell, Bertrand. 1904. On denoting. Mind 14. 479-493.

Schlenker, Philippe. 2012. "Maximize Presupposition" and Gricean reasoning. Natural Language Semantics 20(4). 391-429. doi:10.1007/s11050-012-9085-2.

Spector, Benjamin. to appear. Comparing exhaustivity operators (minimal worlds vs. innocent exclusion). Semantics and Pragmatics .

Strawson, Peter Frederick. 1950. On referring. Mind 59. 320-344.

Walker, Ralph C. S. 1975. Conversational implicatures. In S. Blackburn (ed.), Meaning, reference, and necessity: new studies in semantics, Cambridge, England: Cambridge University Press.

Giorgio Magri

SFL UMR 7023

CNRS and University of Paris 8

magrigrg@gmail.com 